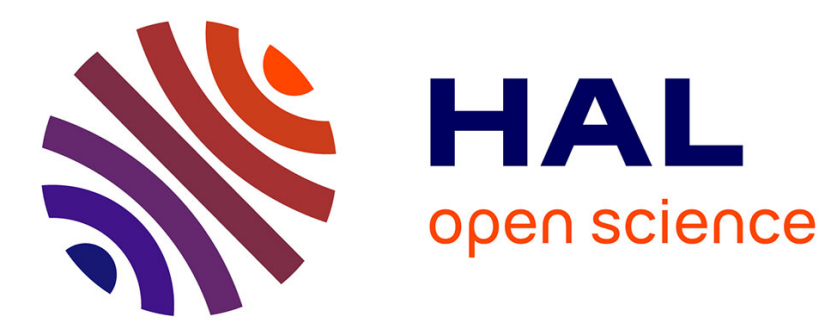

\title{
The sense of social agency in gaze leading
}

\author{
Samuel Recht, Ouriel Grynszpan
}

\section{To cite this version:}

Samuel Recht, Ouriel Grynszpan. The sense of social agency in gaze leading. Journal on Multimodal User Interfaces, 2019, 12 (38), pp.1-12. 10.1007/s12193-018-0286-y . hal-01977395

\section{HAL Id: hal-01977395 \\ https://hal.sorbonne-universite.fr/hal-01977395}

Submitted on 10 Jan 2019

HAL is a multi-disciplinary open access archive for the deposit and dissemination of scientific research documents, whether they are published or not. The documents may come from teaching and research institutions in France or abroad, or from public or private research centers.
L'archive ouverte pluridisciplinaire HAL, est destinée au dépôt et à la diffusion de documents scientifiques de niveau recherche, publiés ou non, émanant des établissements d'enseignement et de recherche français ou étrangers, des laboratoires publics ou privés. 


\section{The sense of social agency in gaze leading}

Abstract: Social interactions entail reciprocal reactions where one's communicative acts triggers responses in others. Fluent interpersonal exchange relies on the ability to discriminate behaviors produced by others that are responses to one's actions, thus involving a social sense of agency. Given the pivotal role of gaze in human communication, we propose to use gaze following as a model for studying the sense of agency in social actions. The experiment investigates the influence of sensory expertise and timing of the action's effects by comparing feedback provided by a human avatar versus a nonfigurative animated object (an arrow) and by varying the control exerted by participants' gaze on the feedback (avatar vs arrow). Results revealed a linear relationship between the judgement of agency and feedback latencies and higher agency discriminating performances with the avatar. These outcomes suggest that classical cognitive accounts of the sense of agency can be expanded to the realm of social actions and provide important information for designing virtual agents to train social gaze interactions.

Keywords: agency, social cognition, forward model, eye-tracking, avatar 


\section{Introduction}

\subsection{Social agency}

Social interactions entail reciprocal reactions where one's communicative acts triggers responses in others. The consequences of one's social action on another partaker provides feedback to the former about her/his action. Efficient social communication is achieved when one is able to regulate her/his own behavior on the basis of the feedback received from others. As Bandura [1] reminds us, during interpersonal exchanges, "people are each other's environments" (p. 165). Self-regulation of behavior with respect to others' social reactions appears early in life as illustrated by the functional role of imitation in the development of communicative skills [2]. Pre-verbal infants tend to spontaneously use imitation as a mean to achieve social contingency with peer partners through reciprocal adjustments of behaviors [3]. Deficits in the ability to adjust to the consequences of one's behavior in social contexts have been associated with impairments such as those observed in Autism Spectrum Disorders [4]. As emphasized by Loveland [5], "successful self-regulation requires a rapid assessment of one's own behavior and reactions, as well as their effects on others and the situation" (p. 369) and "persons with autism have special difficulty not only with perceiving social and emotional aspects of the world, but also with using this information to regulate their own behavior and emotions in appropriate ways" (p. 370).

Social reactions often follow routines that can be anticipated, as when one holds out her/his hand and expects the other to grab it and shake it. A lack of the expected responses by a social other can fuel the impression of being ignored and lead to negative feelings towards the other. The ability to identify the consequences of one's social actions thus enables fluent interpersonal communication. Yet, it may be prone to errors, notably because social partakers are autonomous agents that behave according to their own will. For social interactions to run smoothly, one needs to discriminate social responses that are consequences of her/his actions from behaviors that are unconnected. We therefore contend that, to be a social agent, an individual needs to have a sense of which behaviors in others were caused by her/him. 
The present paper investigates the mechanisms that underlie the sense of being an agent in social interactions. The sense of agency refers to the experience of controlling one's own actions and their effects on the world [6]. According to Gallagher [7], it is "The sense that I am the one who is causing or generating an action" (p. 15). He emphasizes how the ability to judge events as self-generated enables us to define who we are. Although the sense of agency is commonly viewed as essential for social functioning [8], it has rarely been investigated in the context of social actions. If the sense of agency has been widely studied for self-generated actions on objects, its extension to social interactions has been largely ignored. Some studies pertained to the influence of social context on the sense of agency when manipulating objects [9] or to the sense of agency in joint actions with others [10], but the causal relationship between one's actions and other people's reactions has only been marginally addressed in this field of research. Pfister, Obhi, Rieger and Wenke [11] reported an experiment where participants developed a sense of agency for another person's behavior that was triggered by their own action. However, participants' actions and their partners' reactions were simple keypresses that were abstractly related due to task demands and did not carry any social meaning, thus contrasting with real life social interactions. In the present work, the sense of social agency refers to the experience of controlling one's own socially meaningful actions and their effects on others.

Transposing the experimental frameworks of the classical agency literature to the social realm requires fine-grained control over the possible expressions of social actions. Manually mediated social interactions, such as handshaking, usually involve joint actions in which movement production is shared and individual responsibilities are hard to disentangle. For this reason, gaze appears to be an interesting experimental model to introduce this new perspective, as eyes have the advantage of yielding clearly separable movements that are relatively simple with limited degrees of freedom. Gaze holds an important role as an action in the realm of social exchanges, which goes beyond its usual perceptive function. Film director Louis Malle illustrates the dreadful impact that gaze can have on the outcome of a 
social encounter in his autobiographical film "Au revoir les enfants" ("Goodbye, children", release date: 1987) about his childhood during World War Two. In the most dramatic scene, he looks at his Jewish friend while the Gestapo is raiding his school, and thus unintentionally draws the attention of the Gestapo officer toward his friend, which gives him away. Eye movements convey meaningful messages that can influence the behavior of people we are socially engaged with. Gaze is one of the active modalities that makes us agents of social interactions. Judgement of agency occurs when we consciously ascribe to ourselves the social consequences of our eye movements, just as Louis Malle took responsibility for the fate of his friend. Experimental evidence shows that we are able to quickly learn to associate our gaze movements with social reactions displayed by others, such as changes in emotional facial expression, and, furthermore, that we then anticipate these changes [12]. Verschoor, Spapé, Biro and Hommel [13] found that spontaneous action - effect binding occurred for eye saccades as early as in the first year of life. The social agency of gaze can thus be experienced from the very early stages of social development.

\subsection{Theoretical accounts of the sense of agency}

During the last twenty years, several theories have been forged to model the sense of agency. A hypothesis classically advanced is the "comparator model". It posits the existence of an internal predictive mechanism, called the "forward model", embedded in the central nervous system [14]. When an action is initiated, the forward model receives a duplicate of the motor signals, so-called the "efference copy", on the basis of which it predicts the sensory outcomes of the action. This prediction is then compared with the actual sensory consequences of the action. The sense of agency results from the congruency between the two. According to Wolpert, Doya and Kawato [15], this model can be extended to social interactions. Research on the human visual system provides evidence supporting the existence of a "forward model" for eye movements and possible neural pathways for the efference copy [16-18]. The concept of "forward model" was first introduced as a building block of sensorimotor control [19, 20]. 
As such, the "forward model" should be tuned to the degree of expertise that an individual has with a given sensorimotor environment. According to the comparator model hypothesis, the sense of agency depends on whether the "forward model" can predict the sensory consequences of actions. Therefore, in this view, the ability to recognize one's agency requires some degree of familiarity with the sensory feedback.

An alternative theory, often referred to as the reconstructionist approach [21], purports that the sense of agency follows similar rules than the general cognitive mechanisms used to deduce causality [22]. In this perspective, an action is self-attributed when the conscious thought of acting preceded the action, the action and the thought were consistent and the action was not better explained by other available options. The sense of agency is thus viewed as a retrospective mechanism. Familiarity with the sensory feedback is of lesser importance in this framework. By contrast, the timing of the feedback plays a major role. Experimental evidence on finger movements shows that the sense of agency decreases when the delay between the action and its effects increases [23-27]. Whether this applies to social actions needs confirmation. Recent literature tries to bridge the gap between the comparator model and the reconstructionist theory $[28,29]$. The goal of the present research was to investigate the influence of timing and sensory expertise on the sense of agency in the case of social gaze movements generated by a virtual human.

\subsection{Gaze following as a model of social agency}

Most of the studies on the sense of agency focus on limb movement [30] and very few have been devoted to gaze $[4,31,32]$. Gaze nevertheless provides an insightful paradigm to investigate the sense of agency in the context of social interactions. The first step of our study was to identify a familiar social feedback of gaze actions. A typical example of gaze movements eliciting reactions in others is gaze following. Gaze following designates the natural tendency to respond to the gaze orientation of another individual by following her/his line of sight. It is considered highly linked to the specific features of the 
human eye acquired through evolution [33]. Gaze following has been extensively studied using an adaptation of Posner's task [34]. It has also been coined the "Gaze Cueing Effect" [35, 36], which emphasizes the deictic action produced by gaze. Evidence suggests that visual attention is automatically prompted by gaze cueing, tantamount to a reflexive mechanism [37, 38]. Gaze following is considered to be an essential element of joint attention [35, 39], which starts emerging around six months of age [40, 41] and forms the basis of our ability to coordinate attention with a social partner [42]. Edwards, Stephenson, Dalmaso and Bayliss [43] recently revealed that one's attention is automatically reoriented towards the eyes of another individual who followed her/his gaze. According to these authors, this mechanism would be instrumental in our ability to monitor the effects of our gaze on others during joint attention episodes. They underline the need to investigate the sense of agency when one leads the gaze of another person and emphasize that "the time course of these interactions will be critical" (p. 6). We thus reasoned that gaze following would represent a relevant naturalistic setting for investigating the sense of social agency in gaze and that such studies would foster better understanding of the mechanisms involved in joint attention.

The present study tested the influence of sensory expertise and feedback delay on the sense of agency in social gaze. We compared a familiar social feedback versus a non-social feedback of eye movements. The familiar social feedback was provided by simulating a human face who followed the gaze of the participant, while the non-social feedback was supplied via a nonfigurative object, that is, an arrow. Using a real human for the familiar social feedback would have come at the expense of controllability, because real humans cannot be expected to control their gaze in the same systematic and timely manner as computers. We therefore designed a human avatar and an arrow that were controlled using the same algorithms. Arrows are attentional stimuli that have been compared to eyes or faces in experiments on gaze cueing using the Posner paradigm [44-51]. Arrows are known to elicit automatic orienting of attention in a similar manner to gaze cues [52]. Therefore, an arrow appeared as an appropriate 
comparison stimulus in our study. To equate the movements of the arrow with the human face, we used a realistic human avatar that was controlled by the same computer system than the arrow. Although arrows and gaze cues yield similar effects on attention, they have been reported to involve different cognitive and neural mechanisms [44, 46-49, 53].

The experiment reported here investigated the sense of agency by using an eye-tracker that coupled the two types of feedback with the gaze of the participants. According to the comparator model account, the forward model of a participant should be more finely tuned to familiar sensory feedback of action than to unfamiliar feedback. Therefore, the sensory prediction of the effects of gaze movements should be more accurate when the feedback is conveyed with the avatar than with the arrow, given that the former simulates the common phenomenon of gaze following, while the latter was created artificially and is very unlikely to happen in real life. Hence, participants should be better at discriminating the effects of their gaze on the avatar compared to the arrow. By contrast, the reconstructionist approach places less emphasis on the ability to produce precise sensory predictions. Hence, according to this second theory the sense of agency should be similar for the two types of feedback. These hypotheses are summarized in Table 1. To evaluate the influence of sensory expertise on the sense of social agency in gaze, we sought to accentuate the difference between the two types of feedback in terms of how relevant they were with respect to the real life experience of participants. It was therefore important to optimize the realism of the human avatar rather than using a schematic representation of a face, as the latter could have ended up creating a feedback for eye movements that was comparatively just as unusual as the arrow. We therefore did not try to match the low-level visual features of the two types of feedback. Besides, as emphasized by Guzzon et al. [46], arrows bear specific geometrical asymmetries that are different from those of the eyes and entail distinct spatial distribution of luminance. It thus seemed pointless to try to suppress perceptual differences between the two types of feedback. Moreover, gaze orientation in real life is not only contingent on eye motion, but involves combined eye 
and head movements. Thus, to simulate common naturalistic gaze following behaviors, the avatar was designed to produce such combined eye-head movements. Gaze contingent avatars have previously been used to investigate the emotional and attentional effects of having one's gaze followed by another person, but they usually relied on faces that could only produce a given set of pre-defined movements involving only the eyes $[32,43,54-61]$. Here, we sought to enhance the ecological validity of the stimulus by simulating more natural gaze movements.

Daprati et al. [62] have shown that performances in discriminating self-generated from external actions depends on the congruency of the movement that is fed back. They asked participants to recognize whether hand movements shown to them were theirs or not. Participants could trivially answer when the hand movement they watched was incongruent with the one they generated, but their performances degraded when the movement was congruent. They then had to rely on subtle discrepancies in timing and kinematic. We employed an equivalent protocol transposed to eye movements. Participants had to judge their agency when the feedback was either controlled by their eyes (self-generated action) or by an external source (externally generated action). In the latter case, the movements of the avatar or the arrow were generated independently by the computer and were either congruent or incongruent with respect to the direction in which participants moved their eyes.

Additionally, we sought to assess the effect of time on the sense of agency in gaze. Series of selfgenerated action conditions were created by varying the latency between the eye movements of the participants and the feedback. Our goal was to characterize the strength of one's impression that another person is following her/his gaze as a function of how fast this other person follows her/his gaze. Our hypothesis is detailed in Table 1. Several studies suggest that the sense of agency is best described by a continuous rather than dichotomous mechanism $[23-25,63]$. Therefore, we opted for a continuous scale rating of the sense of agency. In addition, we measured response times to estimate the effort needed to decide whether the feedback was controlled by oneself or by an external source. 
Table 1: Summary of the hypotheses

\begin{tabular}{|l|l|}
\hline Hypothesis & Expected outcome \\
\hline Supporting the & Participants would experience more control over the effect of their gaze \\
when the feedback is conveyed by the avatar compared to the arrow \\
\hline Supporting the & Participants would experience just as much control over the effect of their \\
reconstructionist account & gaze whether the feedback is conveyed by the avatar or the arrow \\
\hline $\begin{array}{l}\text { Supporting the influence } \\
\text { of effect latency }\end{array}$ & Participants would experience less control over the effect of their gaze \\
\end{tabular}

\section{Method}

\subsection{Participants}

Twenty four participants were recruited for this experiment. This sample size matches the highest sample size in the studies that were included in a recent meta-analysis on the sense of agency [64]. Additionally, we conducted a power analysis based on data reported by Daprati et al. [62] for healthy participants who were shown feedback that was congruent with their finger movements but was actually generated by the experimenter [Cohen's $d=1.19$ ]. The significance threshold was set at 0.05 and the power at 0.9. This power analysis was performed with the G*Power application [65]. It indicated that the sample size should be superior to eight.

Participants were free of any known psychiatric or neurologic symptoms, non-corrected visual or auditory deficits and recent use of any substance that could impede concentration. Their age ranged from 18 to 34 years with a mean of 23.25 [SD = 3.63]. The group comprised 11 women and 13 men. This research was reviewed and approved by the regional ethics committee. Informed consent was obtained from each participant. 


\subsection{Material}

Participants were seated in front of a computer that was equipped with an eye-tracker, model X2-60 from Tobii (www.tobii.com). This eye-tracker remotely detected the direction of the eyes without the need for a chin rest or a helmet. Participants could therefore move their eyes and head freely. The sampling rate of the eye-tracker was $60 \mathrm{~Hz}$. The screen size was $380 \times 215 \mathrm{~mm}^{2}$ with a resolution of $1920 \times 1080$ pixels. Participants were placed at an approximate 60-65 cm distance from the screen, in compliance with the eye-tracker's specifications. Either an avatar or an arrow were displayed on the screen. A toolkit called MARC, for Multimodal Affective and Reactive Characters [66], was used to animate the avatar. The 3D model of the avatar was created with the Blender 3D software suite (www.blender.org). To endow the avatar with realistic physical features of a human being, its graphic design was based on a real person (Figure 1). Using the Python programming language and the Tobii Software Development Kit, we developed software that enabled the avatar to follow the gaze of the participant via the eye-tracker. The avatar combined head and eye movements to simulate gaze following. The avatar's movements were designed according to the procedure described by Courgeon, Rautureau, Martin and Grynszpan [67]. The Python language was also used to animate the arrow on the screen (Figure 1), which could also be controlled via the eye-tracker. To drive the movements of the arrow with the same algorithm as the avatar, we needed a reference point on the avatar's face. The reference point that seemed the most adequate was the tip of the nose, because (1) it was the point on the avatar's face that moved with the highest velocity, (2) perception of head orientation is highly dependent on the deviation of the nose [68]. The arrow was therefore designed to match the movements of the tip of the nose of the avatar: It pointed towards the gaze position of the participant on the screen, and when the participant's gaze was in its vicinity, it shortened. The maximum length of the arrow was equal to the maximum displacement of the tip of the nose of the avatar. 


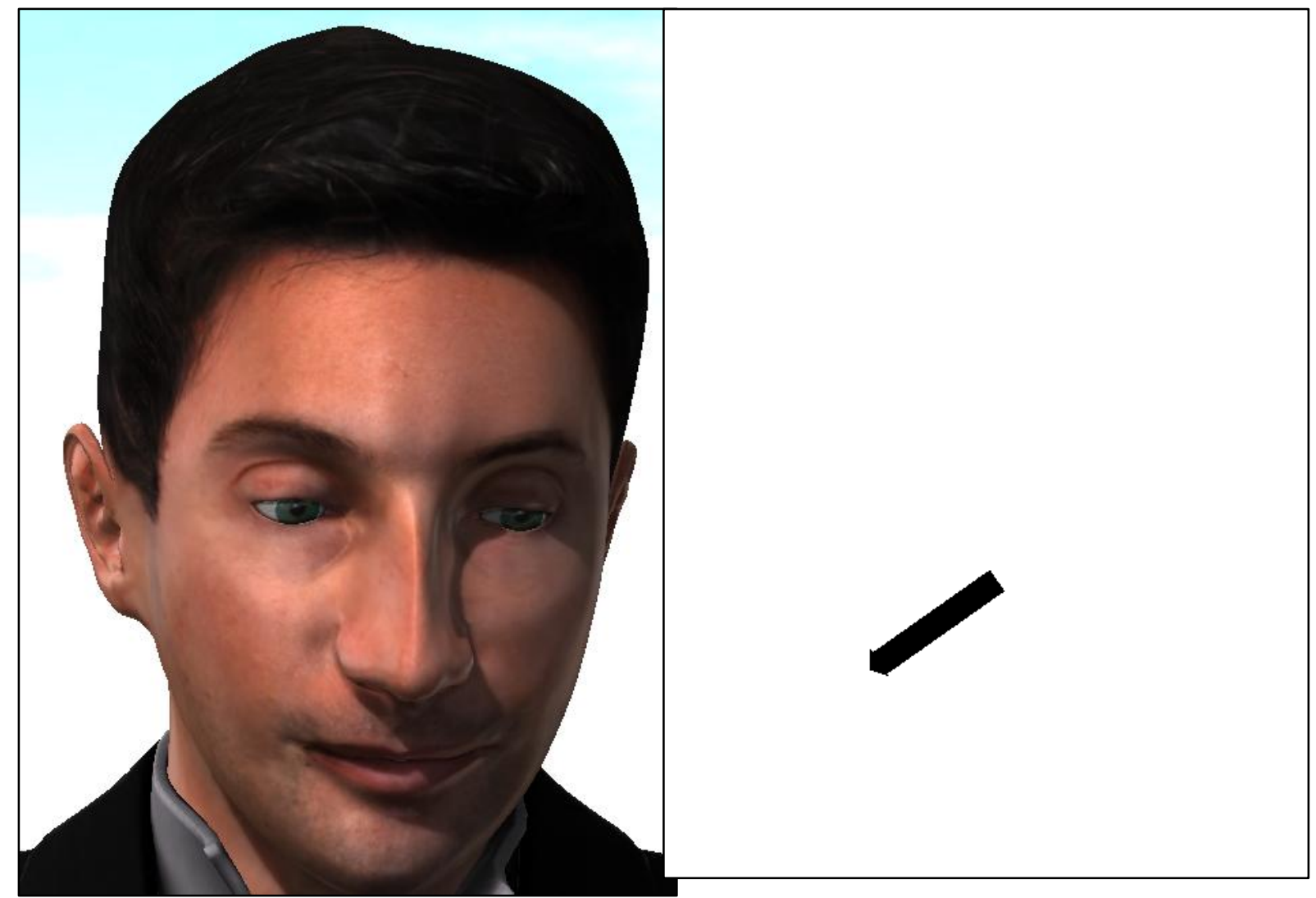

Fig. 1 The graphic models used as visual feedback for the participants' gaze movements. The avatar is displayed on the left and the arrow on the right

\subsection{Procedure}

Two blocks of 80 trials each were administered to the participants. The arrow was displayed during one block, while the human avatar was displayed during the other block. The order of the blocks was randomly counterbalanced across participants. In each trial, the avatar (respectively arrow) was flanked by a " 1 " on one side and a " 2 " on the other side (Figure 2 ). The left or right positions of the " 1 " and " 2 " were allocated randomly. Participants were instructed to first look at " 1 " and then at " 2 ". 


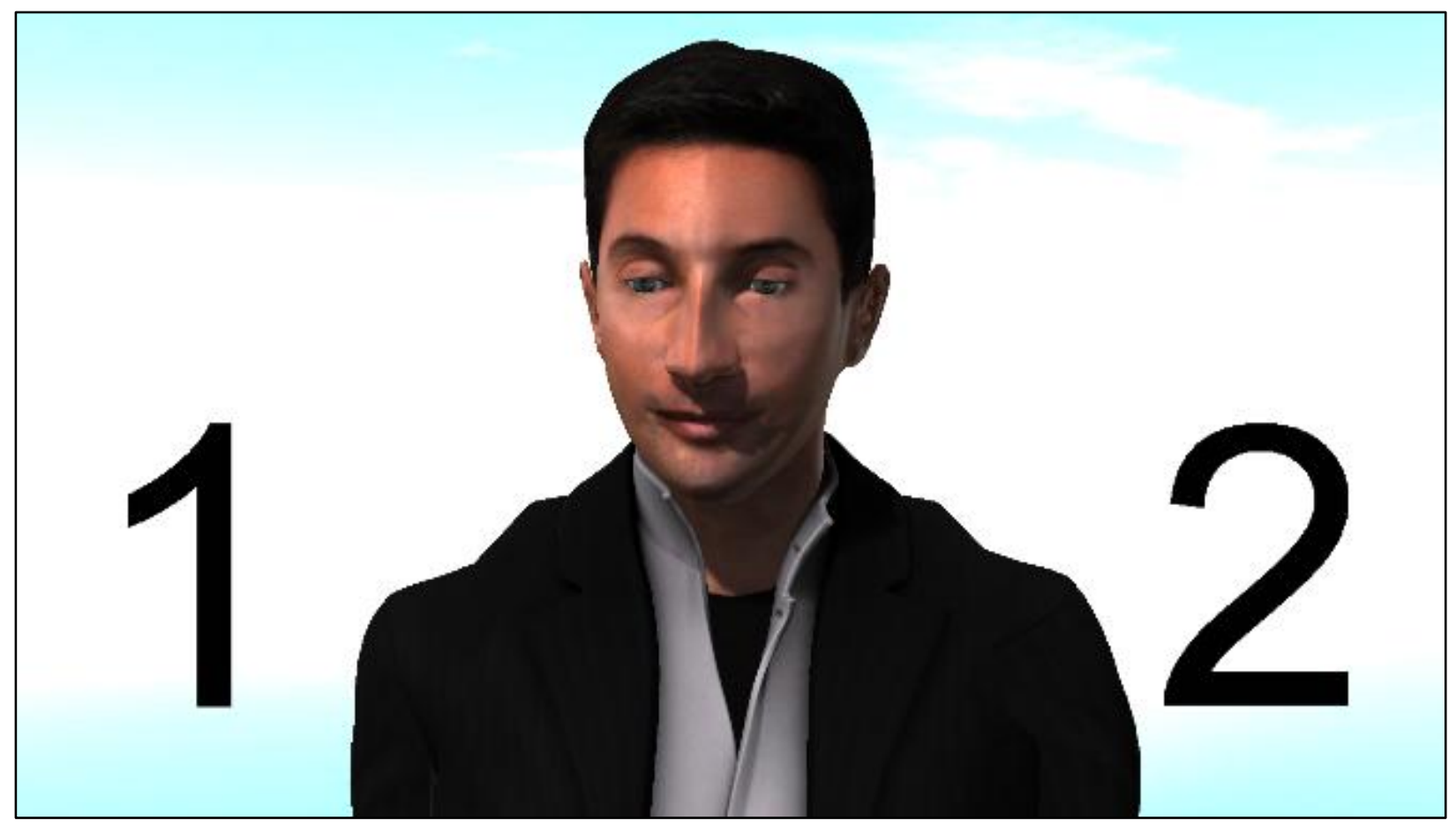

Fig. 2 Screenshots of the avatar flanked by a number " 1 " and a number " 2 " that were randomly positioned on the left or on the right. Participants were instructed to look first at number " 1 " and second at number " 2 ". They then had to rate the extent to which they controlled the avatar with their gaze

The movement of the avatar (respectively the arrow) was programmed to comply with 8 movement conditions that were randomized across trials. Six of these movement conditions involved self-generated actions, that is, the avatar (respectively arrow) was contingent on the eyes of the participant. Those six "self-generated" movement conditions differed with respect to the gaze following latency of the avatar (respectively arrow). The six following latencies were used: 100 ms, 300 ms, 500 ms , 700 ms, 900 ms and 1100 ms. Each trial lasted two seconds. Horowitz et al. [69] report that voluntary shifts of visual attention require between 200 and 300 ms. Looking first at the center of the screen, then at the two numbers and back again at the center required three voluntary shift of visual attention and was thus estimated as taking less than 900 ms. As each trial lasted 2 seconds, participants had enough time to see the reaction of the avatar (respectively arrow) for every latency condition. The initial position of gaze on the center of the screen was enforced by instructing participants to look at a fixation cross that was 
displayed during two seconds before each trial. To avoid erratic movements of the avatar (respectively arrow) due to micro-saccades, the gaze orientation of the avatar (respectively arrow) was derived from the average gaze positions of the participant over a moving window of $100 \mathrm{~ms}$. Hence, the $100 \mathrm{~ms}$ gaze following latency condition could be considered as a quasi-immediate match of the gaze movements of the participant.

The two remaining movement conditions involved externally-generated actions, that is, the gaze orientation of the avatar (respectively arrow) was unrelated to that of the participant. To generate autonomous movements of the avatar (respectively arrow), the system would replay gaze trajectories that had been recorded on a real individual prior to the experiment. In a condition called "externallygenerated congruent", the avatar (respectively arrow) moved in the same direction as the participant, that is, orienting first toward number "1" and then toward number " 2 ", but, given that the movement was pre-recorded, it was not contingent on the participant. In the final condition called "externallygenerated incongruent", the avatar (respectively arrow) followed a pre-recorded trajectory in the opposite direction to the participant, that is, orienting first toward number " 2 " and then toward number “1".

Participants were told that they would be facing an avatar or an arrow that would follow their gaze in some trials and gaze independently in some other trials. They were also informed that there could be latencies in the gaze following movement of the avatar (respectively arrow). After each trial, participants had to rate the extent to which the avatar (respectively arrow) was controlled by their gaze on a Likert scale. They were required to provide a score ranging from 0 to 9 . Zero meant that they had no control at all over the avatar (respectively arrow), while 9 meant that they had full control over the avatar (respectively arrow). Those scores were considered a suitable measure for the sense of agency as they informed on how participants perceived their degree of control over the avatar (respectively arrow). 
Furthermore, to evaluate the difficulty participants had in perceiving their agency, their response time were recorded.

The scores and response times were scanned to remove outliers that were beyond two standard deviations from the mean. Additionally, the Box Cox transform was applied to normalize the data [70]. A two-way repeated measures analysis of variance was conducted using the Greenhouse-Geisser adjustment. The first factor of the ANOVA was the movement condition of the avatar (respectively arrow). As explained above, there were 8 movement conditions: Six of them were "self-generated" movement conditions where the avatar (respectively arrow) was gaze-contingent, that is, it was following the participant's eyes with 6 different latencies $(100 \mathrm{~ms}, 300 \mathrm{~ms}, 500 \mathrm{~ms}, 700 \mathrm{~ms}, 900 \mathrm{~ms}, 1100$ $\mathrm{ms)}$; the two remaining movement conditions were "externally-generated" and were either "congruent" or "incongruent" with respect to the direction in which the participant was instructed to direct her/his gaze. The second factor of the ANOVA represented the graphic model displayed on the screen, that is, either the avatar or the arrow. Post-hoc $t$-tests were performed using the Tukey adjustment procedure. The statistical computations were conducted with the Statistica software (www.statsoft.com).

\subsection{Validation of the stimuli}

Given the potential influence of attentional resources on the agency judgments, we conducted a pilot experiment designed to compare visual detection of the avatar's movements with that of the arrow. This experiment was intended to validate the assumption that the two types of graphic model used in the main experiment were comparable with respect to the accuracy with which participants recognized their movement. The task employed the exact same visual stimuli as those used in the externally generated movement conditions of the main experiment, that is, pre-recorded trajectories that were replayed. In the main experiment, recognizing one's agency involved the ability to appraise the movements of the direction of the avatar's gaze (respectively arrow) with respect to one's own gaze action. In the pilot 
validation experiment, participants were simply required to monitor the orientation of the avatar (respectively arrow) and did not have to give any agency judgment.

\subsubsection{Participants}

A new sample of twenty five participants were recruited for this experiment, so as to obtain consistent sample sizes in the two experiments. Participants were free of any known psychiatric or neurologic symptoms, non-corrected visual or auditory deficits and recent use of any substance that could impede concentration. Their age ranged from 19 to 29 years with a mean of 22.44 [SD $=3.15$ ]. The group comprised 16 women and 9 men. This research was reviewed and approved by the regional ethics committee. Informed consent was obtained from each participant.

\subsubsection{Material}

The setting was similar to the main experiment, except for the eye-tracker that was not needed. The animations obtained from the recordings used in the main experiment served as the original stimuli. In these animations, the avatar (respectively the arrow) first gazed at the center of the screen, then looked at the numbers 1 and 2 in a random order and back at the center of the screen. Animations of more than 2 seconds were generated by aggregating randomly selected recordings. In the resulting animations, the orientation of the avatar (respectively the arrow) could alternate between numbers " 1 " and " 2 " more than once.

\subsubsection{Procedure}

As in the main experiment, a fixation cross was displayed during two seconds before each trial. During the trial, participants watched the animated avatar (respectively arrow) flanked by the " 1 " and " 2 " numbers and had to subsequently determine as fast as possible which of the two numbers was last cued by the avatar (respectively arrow). The duration of each trial varied randomly between 1 and 3 seconds so that participants would not be able to predict the last movement of the avatar (respectively the 
arrow). There were 20 trials in total: 10 for the avatar and 10 for the arrow. The order of the trials was randomly counterbalanced across participants. Participants' success rates in determining the last number cued by the avatar (respectively arrow) and their response times were collected.

As the task was rather simple, the success rates were generally high and their distribution could not be normalized. We therefore used Wilcoxon paired-samples tests to compare the avatar with the arrow under the three movement conditions. The response times were scanned to remove outliers that were beyond two standard deviations from the mean and compared using a paired $t$-test.

\subsubsection{Results and Discussion}

The Wilcoxon tests on success rates yielded no significant differences between the avatar (mean $=0.89$ $S D=0.17)$ and the arrow (mean $=0.85 S D=0.19)[Z=0.99 p=0.32]$. There were no significant differences between the response times of the avatar (mean $=1694 \mathrm{~ms} S D=222 \mathrm{~ms}$ ), and the arrow (mean $=1715 \mathrm{~ms} S D=287 \mathrm{~ms})[t(24)=0.37 p=0.71]$. Results support the assumption that participants had similar performances in detecting movements of the avatar and the arrow.

\section{Results of the main experiment}

The analysis of variance for scores yielded significant main effects for the graphic model $[F(1,23)=12.02$ $\left.p=0.002 \eta^{2}=0.34\right]$ and for the movement condition $\left[F(7,161)=147.34 p<.0001 \eta^{2}=0.86\right]$. With respect to the graphic model, the arrow yielded higher scores than the avatar. Post-hoc tests for the movement conditions showed that scores for "self-generated" movement with gaze following latencies of 100 and 300 ms were significantly higher than for all larger latencies [all $p<.005$ ]. A significant stepwise decrease was observed from 500 to 900 ms latencies [all $p<.0001$ ]. Scores in the "externallygenerated incongruent" condition were lower than scores in the "externally-generated congruent" condition $[p<0.0001]$. They were also lower than in "self-generated" movement conditions with gaze following latencies equal or below $900 \mathrm{~ms}$ [all $p<.0001$ ]. Scores in the "externally-generated congruent" 
condition were significantly lower than in "self-generated" movement conditions for latencies of 100 and $300 \mathrm{~ms}$ [all $p<.0001$ ], but they were higher when the latencies were larger or equal to $700 \mathrm{~ms}$ [all $p<.005]$.

There was also a significant interaction between the graphic model and the movement condition $\left[F(7,161)=4.90 p<.001 \eta^{2}=0.18\right]$. Post hoc tests showed that the "externally-generated congruent" condition was the only condition where scores were significantly higher with the arrow than with the avatar [ $p<.0001]$ (Figure 3). With the arrow, the "externally-generated congruent" condition yielded significantly lower scores than "self-generated" movement conditions when the gaze following latencies were smaller or equal to 300 ms and significantly higher scores when the latencies were above or equal to $700 \mathrm{~ms}$ [all $p<0.0001$ ]. By contrast, with the avatar, scores in the "externally-generated congruent" condition were significantly lower than scores in the "self-generated" movement conditions for latencies smaller or equal to $500 \mathrm{~ms}$, and they were higher for latencies above or equal to $900 \mathrm{~ms}$ [all $p<.0001$ ]. 


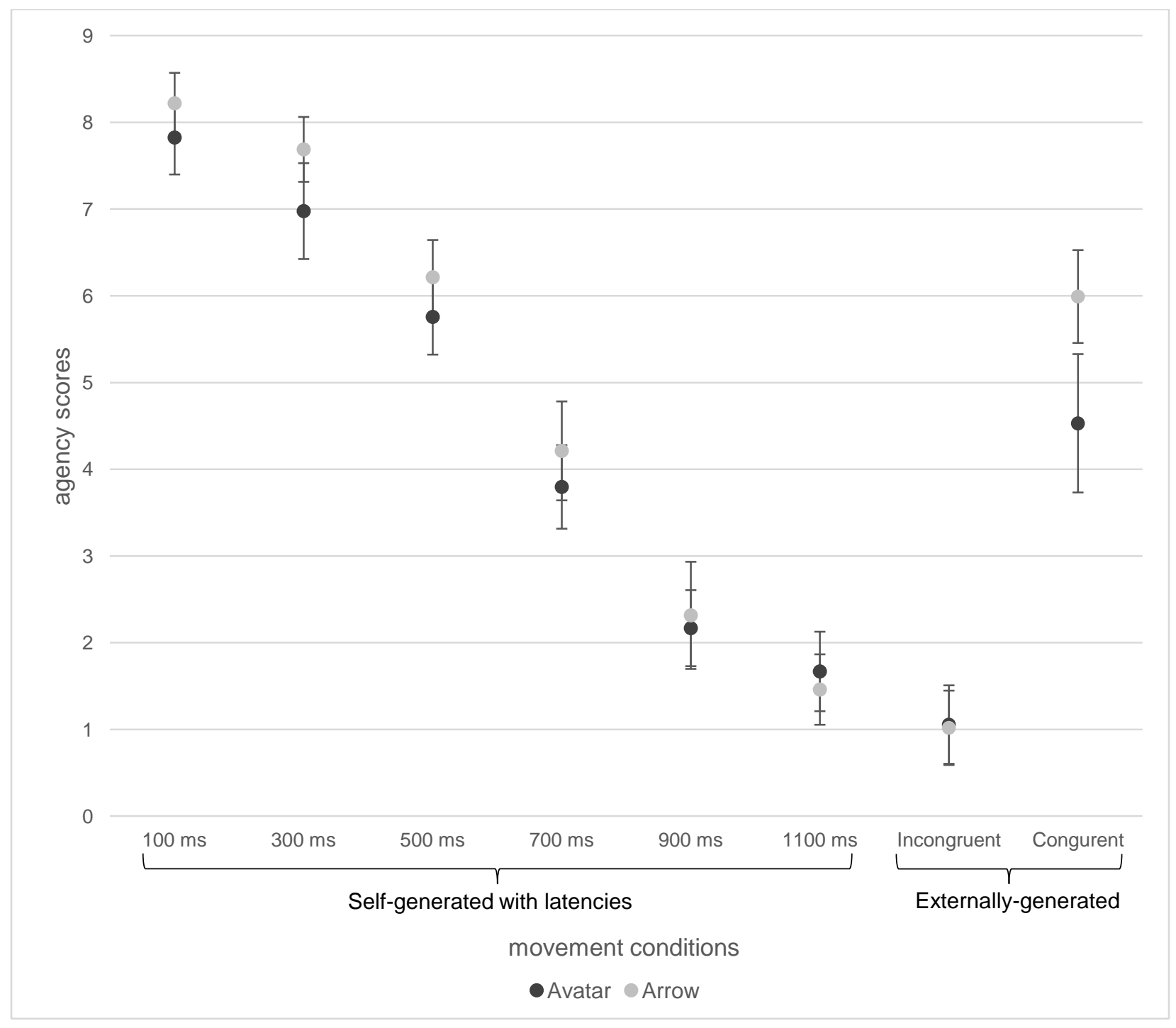

Fig. 3 Scores rating the judgement of agency according to movement conditions when participants faced either the avatar or the arrow. The movement conditions on the X axis were: Six different "self-generated" movement with gaze following latencies ranging from 100 ms to 1100 ms; two "externally-generated" movement that were either "incongruent" or "congruent" with respect to the direction in which participants were instructed to direct their gaze. Error bars represent confidence intervals

To estimate the relationship between the scores and the latencies for "self-generated" movement conditions, that is, when the avatar (respectively arrow) followed the gaze of the participant, we 
conducted a linear regression analysis with the six latencies ranging from $100 \mathrm{~ms}$ to $1100 \mathrm{~ms}$. The result yielded an adjusted $R^{2}=0.82$ for the avatar and $R^{2}=0.84$ for the arrow (Figure 4).

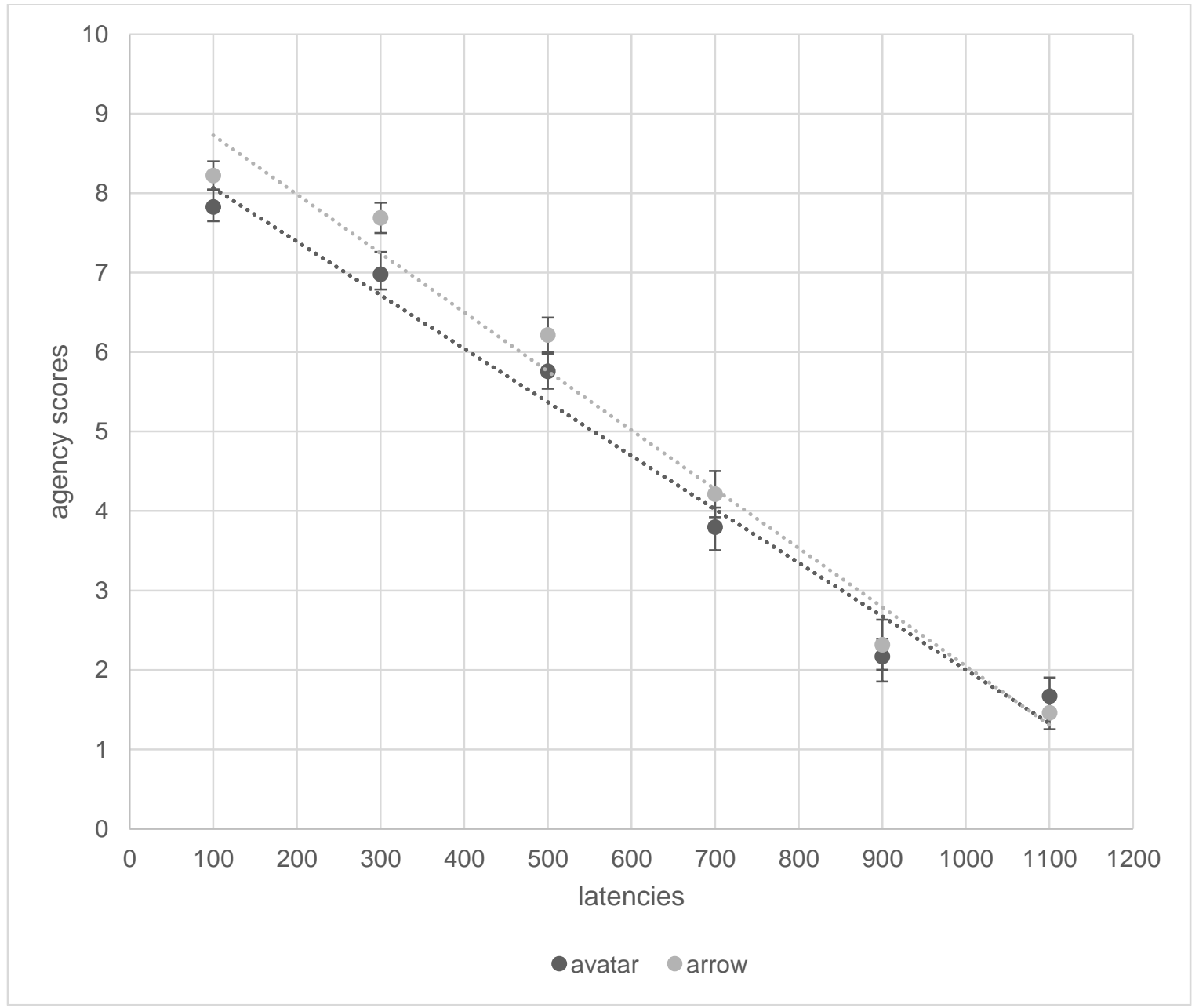

Fig. 4 The relationship between scores for the sense of agency and latencies of gaze following movements for the avatar (respectively the arrow) in the self-generated movement conditions. A regression analysis showed that this relationship was well approximated by a linear function. Data points are the mean scores for each latency and error bars are the standard errors. The dotted lines represent fitted linear functions

The analysis of variance for response time yielded significant main effects for the graphic model $\left[F(1,23)=20.03 p<.001 \eta^{2}=0.47\right]$ and the movement condition $\left[F(7,161)=4.90 p<.001 \eta^{2}=0.18\right]$. Response times were longer for the avatar than the arrow. Post hoc tests on "self-generated" movement conditions showed that response times for the $100 \mathrm{~ms}$ gaze following latency were significantly shorter 
than for latencies of 500, 700 and $900 \mathrm{~ms}$ [all $p<.01$ ]. Response times in the "externally-generated incongruent" condition were significantly shorter than in "self-generated" movement conditions with gaze following latencies of 500 and $700 \mathrm{~ms}$ [all $p<.03$ ]. The interaction between the graphic model and the movement condition was not significant.

\section{Discussion}

As we had hypothesized, when the avatar (respectively arrow) was contingent on the gaze of the participants, scores for the judgement of agency decreased as the gaze following latency increased. The present experiment revealed a linear relationship between the rating scores for agency and the latencies in gaze following movements. This finding that was equally true for the avatar and the arrow is consistent with the view that the sense of agency relies on causal inferences between actions and effects. It is thus consistent with the reconstructionist approach. It does not contradict the comparator model account either, even if the forward model was less well tuned to the arrow because it was an unfamiliar feedback. Research on ideomotor learning shows that the association between eye movements and their sensory effects can be rapidly acquired [13]. Hence, as the experiment unfolded, the forward model could have been adjusting its prediction of the arrow's movements, even if it was initially unfamiliar with this highly unusual feedback for eye movements. This "on the fly" learning process provides an explanation, that is consistent with the comparator model, of why agency scores showed a similar trend for the arrow and for the avatar when gaze movement latencies increased. Notwithstanding, the type of feedback did modulate the judgement of agency, as suggested by the main effect of the graphic model: The arrow was scored higher than the avatar and participants were slower to respond when facing the avatar. Moreover, there was an interaction between the graphic model and the movement conditions for agency scores. When the movement of the avatar (respectively arrow) was externally generated, that is, the computer replayed a pre-recorded trajectory that was unrelated to the gaze of the participant, the rating of agency yielded distinct results depending on whether the avatar's 
movement (respectively arrow) was congruent or incongruent with the eye movement of the participant. As expected, when the movement of the avatar (respectively arrow) was incongruent, that is, in the direction opposite to the gaze of the participant, the agency was trivially judged as externally caused, as shown by very low scores. The answers were not as straightforward when the movement of the avatar (respectively arrow) was congruent, that is, in the same direction as the gaze of the participant. In this condition, the agency ratings were significantly higher for the arrow than for the avatar. Therefore, when the ambiguity between externally-generated actions and self-generated actions was high due to movement congruity, participants were better at discriminating external agency from their own self-agency when they were facing the avatar compared to the arrow. This higher performance can be explained by participants' expertise in detecting gaze following on human faces and thus provides evidence in favor of the comparator model. We thus conclude that the outcome supports the first hypothesis of Table 1.

Alternatively, the face of the avatar being larger than the arrow, one could argue that the movement of the former was easier to perceive than the movement of the latter. However, the movement of the avatar was distributed over the eyes and the head, therefore head motion alone was not enough to grasp the entire gaze movement. Moreover, the arrow was colored black on a white background, thus yielding a high contrast that was easily detectable. As explained earlier, the length of the arrow matched the extension of the displacement of the tip of the nose of the avatar. Given that the nose is a major cue for head orientation [68] and that the tip of the nose was the feature of the avatar's face with the highest movement magnitude, the arrow can be considered as yielding comparable, if not simpler, visual information regarding gaze movement than the avatar. Additionally, this issue of motion recognition was tentatively addressed in the pilot experiment that we conducted to compare the two stimuli. This pilot experiment showed comparable performances for the avatar and the arrow on a simple motion detection task. 
Response times in this first experiment were longer when the participant was exposed to the avatar compared to the arrow. This result suggests that attributing agency to oneself versus to an external source was harder when facing the avatar than the arrow. The fact that response times were slower with the avatar is not well explained by either of the two explanatory theories introduced earlier for the sense of agency. In the reconstructionist approach, the causal inferences are the same for the two stimuli and should take the same amount of time. In the comparator model, the less accurate prediction generated by the "forward model" in the case of the arrow should make the decision process more difficult and therefore longer than for the avatar. Hence, the comparator model predicts the exact opposite of what was observed. Gaze and arrow cues are known to elicit automatic orienting of visual attention [37, 38, 52], but yet differ in how they involve social cognitive processes [53]. Such differences could account for the difference in processing time that were found in the present experiment.

In the present study, we attempted to simulate a real life situation where judging one's agency matters. Obviously, when facing a social partner, one needs to know whether or not she/he is the cause of the partner's change of gaze orientation. It is a necessary condition for joint attention and can have social implications. Therefore, the personal stakes are higher than in typical laboratory experiments on the sense of agency that rely on senseless actions on physical objects such as keypresses. To approach real life settings, we used a realistic human avatar. However, as it was controlled by a computer program, participants may have been less receptive to the social relevance of its gaze behavior than if it had been a true person. An extension of the present study could be to examine the sense of agency when participants are driven to believe that the avatar is controlled by a real person. Additionally, as we sought to simulate naturalistic behaviors, we designed an avatar that combined eye and head movements. An interesting future addition to the present work would be to test whether the results reported here would remain if only the eyes of the avatar could move. Avatars on 2D displays are commonly associated with an illusion, called the Mona Liza effect, whereby observers perceive the 
avatar's eyes as following them when they move around it, although the avatar's eyes are actually steady [71]. This illusion is unlikely to explain the outcomes of the present study as participants remained seated in the same position throughout the experiment. Moreover, the avatar's movement was not restrained to the eyes, but also involved the entire head. The Mona Lisa effect has been reported to disappear in 3D projection surfaces [71]. Gaze direction detection may therefore be more accurate in such settings. Our paradigm could be transposed to 3D virtual reality or with robots to test the influence of these settings on the sense of agency. During joint attention episodes, sensorimotor expertise and social attentional skills could prove to be significant assets for determining agency. Future studies should investigate if the sense of social agency can influence joint attention abilities as suggested by Edwards et al. [43].

Using gaze contingent human avatars, Pfeiffer et al. [32] had already noticed an effect of congruency and latency of eye movements on the judgement of agency, but their results yielded a peak in ratings of selfagency when the gaze following latency was equal to $400 \mathrm{~ms}$. However, their avatar could only produce two types of horizontal eye movements and the delays in their experiments varied in steps of $400 \mathrm{~ms}$. These technological and methodological differences could explain the discrepancies between their study and ours. More specifically, when the gaze following latency was equal to $0 \mathrm{~ms}$ in the study of Pfeiffer et al. [32], participants' gaze movements may have interfered with their ability to pay attention to the small displacement of the iris of the avatar, which could account for lower agency scores compared to the 400 ms latency. By contrast, the avatar in our experiment displayed large movements, involving the head and the eyes, which were easier to attend to. Additionally, Pfeiffer et al. [32] assessed the sense of agency by asking participants to rate "how strongly related they experienced the gaze reaction of the other to their own gaze shift" (p. 4). The sense of agency entails one's causality, which is not necessarily the case of relatedness, so these two notions cannot be equated. Hence, the study of Pfeiffer et al. [32] and ours also differed in this respect. 
Our first experiment showed that the magnitude of the judgement of agency on a continuous scale decreased proportionally with latencies. The data curves reported by Kawabe et al. [24] or Sato and Yasuda [25], who employed continuous scales for rating the sense of agency of finger movements, seem to follow a linear decreasing trend quite similar to that found in the present study. The linear relationship between the judgement of agency and feedback latencies could therefore apply not only to gaze movements, but also to other sensorimotor modalities. In the context of gaze, this relationship constrains social gaze interactions: Gaze following reactions that occur too late could be misattributed and thus impede social exchanges.

The present study illustrated how theoretical frameworks developed in the classical literature on the sense of agency can be transposed to the social realm. Additionally, the outcomes point toward the contribution of specifically social cognitive mechanisms, such as sensorimotor expertise in interpersonal exchange. These contributions underscore the distinctive features of the sense of agency for social actions directed at others, as compared to the classical sense of agency for physical actions on objects. The sense of social agency thus appears to be a relevant construct that could shed light on social cognitive functioning. A reasonable assumption is that mental state attribution depends on the ability to analyze the reactions of others and discriminate those that are responses to one's social actions from those that are independent behaviors guided by an autonomous will. The sense of social agency can thus be conceived as a building block of theory of mind, which would be specifically relevant to live social interactions. Further research on the sense of social agency could enhance our understanding of what some authors call "spontaneous mental state attribution" [72], which is hard to grasp in laboratory settings, although it is common in real life social communication and is considered critical in Autism Spectrum Disorders (ASD). Senju, Tojo, Dairoku and Hasegawa [73] reported an experiment where children with autism did not show preferential sensitivity to gaze cues compared to arrow cues whereas typically developing children did. It would therefore be worth adapting our experimental paradigm to 
analyze sensorimotor expertise of gaze following movements in ASD. Finally, the linear relationship between agency scores and the latencies in gaze following movements found in the present study provides important information on time parameters for those who wish to design artificial agents that follow the user's gaze. Artificial agents such as robots have been used to train joint attention skills in individuals with ASD [74]. Improving gaze following latencies in these robots may enhance their efficiency.

\section{Compliance with Ethical Standards}

Funding: This study was funded by the Labex SMART (ANR-11-LABX-65) supported by French state funds managed by the ANR within the Investissements d'Avenir programme under reference ANR-11-IDEX0004-02 and by La Fondation Orange (71/2012).

Conflict of Interest: The authors declare that they have no conflict of interest.

Data availability: The datasets generated during and/or analyzed during the current study are available from the corresponding author on reasonable request. 


\section{References}

1. Bandura A (2006) Toward a Psychology of Human Agency. Perspect Psychol Sci 1:164-180 . doi: 10.1111/j.1745-6916.2006.00011.x

2. Nadel J, Guérini C, Pezé A, Rivet C (1999) The evolving nature of imitation as a format for communication. In: Imitation in Infancy. Cambridge University Press, New York NY, US, pp 209-234

3. Nadel J (2002) Imitation and imitation recognition: Functional use in preverbal infants and nonverbal children with autism. In: The imitative mind: Development, evolution, and brain bases, eds AN Meltzoff, W Prinz. Cambridge, UK: Cambridge University Press, pp 42-62

4. Grynszpan O, Nadel J, Martin J-C, et al (2012) Self-monitoring of gaze in high functioning autism. J Autism Dev Disord 42:1642-1650 . doi: 10.1007/s10803-011-1404-9

5. Loveland KA (2005) Social-emotional impairment and self-regulation in autism spectrum disorders. In: Nadel J, Muir D, editors. Emotional Development: Recent Research Advances,. Oxford University Press, UK, pp 365-382

6. Haggard P, Chambon V (2012) Sense of agency. Curr Biol 22:R390-R392 . doi: 10.1016/j.cub.2012.02.040

7. Gallagher S (2000) Philosophical conceptions of the self: implications for cognitive science. Trends Cogn Sci 4:14-21 . doi: 10.1016/S1364-6613(99)01417-5

8. Jeannerod M (2003) The mechanism of self-recognition in humans. Behav Brain Res 142:1-15

9. van der Wel RPRD, Sebanz N, Knoblich G (2012) The sense of agency during skill learning in individuals and dyads. Conscious Cogn 21:1267-1279 . doi: 10.1016/j.concog.2012.04.001

10. Obhi SS, Hall P (2011) Sense of agency and intentional binding in joint action. Exp Brain Res 211:655-662 . doi: 10.1007/s00221-011-2675-2

11. Pfister R, Obhi SS, Rieger M, Wenke D (2014) Action and perception in social contexts: intentional binding for social action effects. Front Hum Neurosci 8: . doi: 10.3389/fnhum.2014.00667

12. Herwig A, Horstmann G (2011) Action-effect associations revealed by eye movements. Psychon Bull Rev 18:531-537 . doi: 10.3758/s13423-011-0063-3

13. Verschoor SA, Spapé M, Biro S, Hommel B (2013) From outcome prediction to action selection: developmental change in the role of action-effect bindings. Dev Sci 16:801-814 . doi: 10.1111/desc.12085

14. Blakemore SJ, Wolpert DM, Frith CD (2002) Abnormalities in the awareness of action. Trends Cogn Sci 6:237-242

15. Wolpert DM, Doya K, Kawato M (2003) A unifying computational framework for motor control and social interaction. Philos Trans R Soc Lond B Biol Sci 358:593-602 . doi: 10.1098/rstb.2002.1238 
16. Sperry RW (1950) Neural basis of the spontaneous optokinetic response produced by visual inversion. J Comp Physiol Psychol 43:482-489

17. von Holst E, Mittelstaedt H (1950) Das Reafferenzprinzip. Naturwissenschaften 37:464-476 . doi: 10.1007/BF00622503

18. Wurtz RH (2008) Neuronal mechanisms of visual stability. Vision Res 48:2070-2089

19. Wolpert DM, Ghahramani Z, Jordan MI (1995) An internal model for sensorimotor integration. Science 269:1880-1882

20. Wolpert DM, Miall RC (1996) Forward Models for Physiological Motor Control. Neural Netw Off J Int Neural Netw Soc 9:1265-1279

21. Haggard P (2005) Conscious intention and motor cognition. Trends Cogn Sci 9:290-295 . doi: 10.1016/j.tics.2005.04.012

22. Wegner DM (2003) The mind's best trick: how we experience conscious will. Trends Cogn Sci 7:6569 . doi: 10.1016/S1364-6613(03)00002-0

23. Farrer C, Valentin G, Hupé JM (2013) The time windows of the sense of agency. Conscious Cogn 22:1431-1441 . doi: 10.1016/j.concog.2013.09.010

24. Kawabe T, Roseboom W, Nishida S (2013) The sense of agency is action-effect causality perception based on cross-modal grouping. Proc R Soc Lond B Biol Sci 280:20130991 . doi: 10.1098/rspb.2013.0991

25. Sato A, Yasuda A (2005) Illusion of sense of self-agency: discrepancy between the predicted and actual sensory consequences of actions modulates the sense of self-agency, but not the sense of self-ownership. Cognition 94:241-255 . doi: 10.1016/j.cognition.2004.04.003

26. Shanks DR, Dickinson A (1991) Instrumental judgment and performance under variations in actionoutcome contingency and contiguity. Mem Cognit 19:353-360 . doi: 10.3758/BF03197139

27. Shanks DR, Pearson SM, Dickinson A (1989) Temporal contiguity and the judgement of causality by human subjects. Q J Exp Psychol Sect B 41:139-159 . doi: 10.1080/14640748908401189

28. Moore JW, Fletcher PC (2012) Sense of agency in health and disease: a review of cue integration approaches. Conscious Cogn 21:59-68 . doi: 10.1016/j.concog.2011.08.010

29. Synofzik M, Vosgerau G, Newen A (2008) Beyond the comparator model: a multifactorial two-step account of agency. Conscious Cogn 17:219-239 . doi: 10.1016/j.concog.2007.03.010

30. David N, Newen A, Vogeley K (2008) The "sense of agency" and its underlying cognitive and neural mechanisms. Conscious Cogn 17:523-534 . doi: 10.1016/j.concog.2008.03.004

31. Grynszpan O, Simonin J, Martin J-C, Nadel J (2012) Investigating social gaze as an action-perception online performance. Front Hum Neurosci 6:94 . doi: 10.3389/fnhum.2012.00094 
32. Pfeiffer UJ, Schilbach L, Jording M, et al (2012) Eyes on the mind: investigating the influence of gaze dynamics on the perception of others in real-time social interaction. Front Psychol 3:537 . doi: 10.3389/fpsyg.2012.00537

33. Kobayashi $\mathrm{H}$, Kohshima S (2001) Unique morphology of the human eye and its adaptive meaning: comparative studies on external morphology of the primate eye. J Hum Evol 40:419-435 . doi: 10.1006/jhev.2001.0468

34. Posner MI (1980) Orienting of attention. Q J Exp Psychol 32:3-25 . doi: 10.1080/00335558008248231

35. Frischen A, Bayliss AP, Tipper SP (2007) Gaze cueing of attention: visual attention, social cognition, and individual differences. Psychol Bull 133:694-724 . doi: 10.1037/0033-2909.133.4.694

36. Lachat F, Conty L, Hugueville L, George N (2012) Gaze Cueing Effect in a Face-to-Face Situation. J Nonverbal Behav 36:177-190 . doi: 10.1007/s10919-012-0133-x

37. Driver J (1999) Gaze Perception Triggers Reflexive Visuospatial Orienting. Vis Cogn 6:509-540 . doi: $10.1080 / 135062899394920$

38. Friesen CK, Kingstone $A$ (1998) The eyes have it! Reflexive orienting is triggered by nonpredictive gaze. Psychon Bull Rev 5:490-495 . doi: 10.3758/BF03208827

39. Emery NJ (2000) The eyes have it: the neuroethology, function and evolution of social gaze. Neurosci Biobehav Rev 24:581-604 . doi: 10.1016/S0149-7634(00)00025-7

40. Charman T (2003) Why is joint attention a pivotal skill in autism? Philos Trans R Soc Lond B Biol Sci 358:315-324 . doi: 10.1098/rstb.2002.1199

41. Scaife M, Bruner JS (1975) The capacity for joint visual attention in the infant. Nature 253:265-266 . doi: $10.1038 / 253265 \mathrm{a} 0$

42. Mundy P, Newell L (2007) Attention, Joint Attention, and Social Cognition. Curr Dir Psychol Sci Wiley-Blackwell 16:269-274 . doi: 10.1111/j.1467-8721.2007.00518.x

43. Edwards SG, Stephenson LJ, Dalmaso M, Bayliss AP (2015) Social orienting in gaze leading: a mechanism for shared attention. Proc R Soc B 282:20151141 . doi: 10.1098/rspb.2015.1141

44. Brignani D, Guzzon D, Marzi CA, Miniussi C (2009) Attentional orienting induced by arrows and eyegaze compared with an endogenous cue. Neuropsychologia 47:370-381 . doi: 10.1016/j.neuropsychologia.2008.09.011

45. Friesen CK, Ristic J, Kingstone A (2004) Attentional Effects of Counterpredictive Gaze and Arrow Cues. J Exp Psychol Hum Percept Perform 30:319-329 . doi: 10.1037/0096-1523.30.2.319

46. Guzzon D, Brignani D, Miniussi C, Marzi CA (2010) Orienting of attention with eye and arrow cues and the effect of overtraining. Acta Psychol (Amst) 134:353-362 . doi:

10.1016/j.actpsy.2010.03.008 
47. Hietanen JK, Leppänen JM, Nummenmaa L, Astikainen $P$ (2008) Visuospatial attention shifts by gaze and arrow cues: An ERP study. Brain Res 1215:123-136 . doi: 10.1016/j.brainres.2008.03.091

48. Hietanen JK, Nummenmaa L, Nyman MJ, et al (2006) Automatic attention orienting by social and symbolic cues activates different neural networks: An fMRI study. Neurolmage 33:406-413 . doi: 10.1016/j.neuroimage.2006.06.048

49. Marotta A, Lupiáñez J, Martella D, Casagrande M (2012) Eye gaze versus arrows as spatial cues: Two qualitatively different modes of attentional selection. J Exp Psychol Hum Percept Perform 38:326-335 . doi: 10.1037/a0023959

50. Stevens SA, West GL, Al-Aidroos N, et al (2008) Testing whether gaze cues and arrow cues produce reflexive or volitional shifts of attention. Psychon Bull Rev 15:1148-1153 . doi:

10.3758/PBR.15.6.1148

51. Tipples J (2008) Orienting to counterpredictive gaze and arrow cues. Percept Psychophys 70:77-87 . doi: 10.3758/PP.70.1.77

52. Tipples J (2002) Eye gaze is not unique: Automatic orienting in response to uninformative arrows. Psychon Bull Rev 9:314-318 . doi: 10.3758/BF03196287

53. Hooker $\mathrm{Cl}$, Paller KA, Gitelman DR, et al (2003) Brain networks for analyzing eye gaze. Cogn Brain Res 17:406-418 . doi: 10.1016/S0926-6410(03)00143-5

54. Bayliss AP, Murphy E, Naughtin CK, et al (2013) "Gaze leading": initiating simulated joint attention influences eye movements and choice behavior. J Exp Psychol Gen 142:76-92 . doi: $10.1037 / \mathrm{a} 0029286$

55. Dalmaso M, Edwards GS, Bayliss AP (2016) Re-encountering individuals who previously engaged in joint gaze modulates subsequent gaze cueing. J Exp Psychol Learn Mem Cogn 42:271-284 . doi: 10.1037/xIm0000159

56. Dratsch T, Schwartz C, Yanev K, et al (2013) Getting a grip on social gaze: control over others' gaze helps gaze detection in high-functioning autism. J Autism Dev Disord 43:286-300 . doi: 10.1007/s10803-012-1569-x

57. Grynszpan O, Nadel J, Martin JC, Fossati P (in press) The awareness of joint attention: a study using gaze contingent avatars. Interact Stud

58. Kim K, Mundy P (2012) Joint Attention, Social-Cognition, and Recognition Memory in Adults. Front Hum Neurosci 6: . doi: 10.3389/fnhum.2012.00172

59. Oberwelland E, Schilbach L, Barisic I, et al (2016) Look into my eyes: Investigating joint attention using interactive eye-tracking and fMRI in a developmental sample. Neurolmage 130:248-260 . doi: 10.1016/j.neuroimage.2016.02.026

60. Schilbach L, Wilms M, Eickhoff SB, et al (2010) Minds made for sharing: initiating joint attention recruits reward-related neurocircuitry. J Cogn Neurosci 22:2702-2715 . doi:

10.1162/jocn.2009.21401 
61. Wilms M, Schilbach L, Pfeiffer U, et al (2010) It's in your eyes-using gaze-contingent stimuli to create truly interactive paradigms for social cognitive and affective neuroscience. Soc Cogn Affect Neurosci 5:98 . doi: 10.1093/scan/nsq024

62. Daprati E, Franck N, Georgieff N, et al (1997) Looking for the agent: an investigation into consciousness of action and self-consciousness in schizophrenic patients. Cognition 65:71-86

63. Farrer C, Franck N, Georgieff N, et al (2003) Modulating the experience of agency: a positron emission tomography study. Neurolmage 18:324-333 . doi: 10.1016/S1053-8119(02)00041-1

64. Sperduti M, Delaveau P, Fossati P, Nadel J (2011) Different brain structures related to self- and external-agency attribution: a brief review and meta-analysis. Brain Struct Funct 216:151-157 . doi: 10.1007/s00429-010-0298-1

65. Faul F, Erdfelder E, Lang A-G, Buchner A (2007) G*Power 3: A flexible statistical power analysis program for the social, behavioral, and biomedical sciences. Behav Res Methods 39:175-191 . doi: 10.3758/BF03193146

66. Courgeon M, Clavel C (2013) MARC: a framework that features emotion models for facial animation during human-computer interaction. J Multimodal User Interfaces 7:311-319 . doi: $10.1007 / \mathrm{s} 12193-013-0124-1$

67. Courgeon M, Rautureau G, Martin J-C, Grynszpan O (2014) Joint Attention Simulation Using EyeTracking and Virtual Humans. IEEE Trans Affect Comput 5:238-250 . doi: 10.1109/TAFFC.2014.2335740

68. Wilson HR, Wilkinson F, Lin L-M, Castillo M (2000) Perception of head orientation. Vision Res 40:459-472 . doi: 10.1016/S0042-6989(99)00195-9

69. Horowitz TS, Wolfe JM, Alvarez GA, et al (2009) The speed of free will. Q J Exp Psychol 2006 62:2262-2288 . doi: 10.1080/17470210902732155

70. Sakia RM (1992) The Box-Cox transformation technique: a review. The statistician 169-178

71. Al Moubayed S, Edlund J, Beskow J (2012) Taming Mona Lisa : communicating gaze faithfully in 2D and 3D facial projections. ACM Trans Interact Intell Syst 1:25

72. Senju A, Southgate $V$, White $S$, Frith $U$ (2009) Mindblind eyes: an absence of spontaneous theory of mind in Asperger syndrome. Science 325:883-885 . doi: 10.1126/science.1176170

73. Senju A, Tojo Y, Dairoku H, Hasegawa T (2004) Reflexive orienting in response to eye gaze and an arrow in children with and without autism. J Child Psychol Psychiatry 45:445-458

74. Kajopoulos J, Wong AHY, Yuen AWC, et al (2015) Robot-Assisted Training of Joint Attention Skills in Children Diagnosed with Autism. In: Tapus A, André E, Martin J-C, et al (eds) Social Robotics. Springer International Publishing, pp 296-305 